\title{
LOS NIÑOS DEL CAUCA \\ I. DESCRIPCION DE UN FOCO DE RAQUITISMO DEPENDIENTE DE LA VITAMINA D, TIPO II.
}

\author{
Alejandro Giraldo ${ }^{1}$ Wilber Pino ${ }^{2}$ Maritza Pineda ${ }^{3}$ Fernando Garcia ${ }^{4}$ David Freyle ${ }^{5}$, \\ Fernando Nova ${ }^{6}$ Edith Monsalve ${ }^{7}$ Pilar Bernal ${ }^{8}$ Carmelo Arregoces ${ }^{9}$. Antonio Iglesias ${ }^{10}$
}

\begin{abstract}
Al norte del departamento del Cauca se detectó un numeroso grupo de pacientes con deformidades en miembros inferiores (genu varo, genu valgo o ambos) que correspondían clínica y radiológicamente a un cuadro de raquitismo. Ninguno de los pacientes presentaban alopecia, miopatía, tetania o aminoaciduria. La mayoría de ellos proceden de un grupo poblacional semiaislado de ancestro afromestizo, en el corregimiento de La Toma, del municipio de Suárez.
\end{abstract}

Los estudios bioquímicos comparativos entre 64 pacientes y 109 individuos no afectados, parientes en primer grado, mostraron en los pacientes una ligera hipocalcemia, niveles normales altos de fósforo sérico, un sustancial incremento de la fosfatasa alcalina, valores normales de proteínas y albúminas y niveles séricos de paratohormona aumentados. Estudios en orina de 24 horas mostraron hipocalciuria e hipofosfaturia. A un subgrupo de 8 pacientes se les determino niveles séricos de la $25(\mathrm{OH}) \mathrm{D}_{3}$ y l $\alpha, 25(\mathrm{OH}), \Delta_{3}$ para establecer a qué tipo de raquitismo pertenecían nuestros pacientes. Se observó un marcado incremento de $1 \alpha, 25(\mathrm{OH})_{2} \Delta_{3}$, por lo que esta patología corresponde al raquitismo dependiente de la vitamina $\mathrm{D}$, tipo II. Se trata de un tipo de raquitismo genético transmitido en forma autosómica recesiva, cuyas manifestaciones clínicas y de laboratorio se presentan por un defecto en la acción del receptor para la vitamina $D$.

A 115 pacientes se les inició tratamiento con $1 \alpha, 25(\mathrm{OH}){ }_{2} \Delta 3 \mu \mathrm{g} 0,50 /$ día, calcio 1.840 $\mathrm{mg}$ /día y fósforo $1.424 \mathrm{mg} / \mathrm{día}$, administrados en dos dosis diarias. En un grupo de 21 pacientes, se analizaron los datos de laboratorio en suero, pre y posttratamiento un año después y todos los valores tendieron a normalizarse. Estudios moleculares, del mRNA y cDNA del gen del receptor de la vitamina D, obtenidos de cultivo de fibroblastos de dos de los pacientes más severamente afectados, mostraron una secuencia normal de nucleótidos.

Se hace una comparación entre los hallazgos clínicos y de laboratorio entre los 20 casos informados hasta el momento en la literatura con este tipo de enfermedad

\footnotetext{
1 Médico genetista, Director Fundación Arthur Stanley Gillow. Santafé de Bogotá. (1)

2 Médico, Grupo de Genética, Instituto Nacional de Salud

3 Bacterióloga, Grupo de Genética, Instituto Nacional de Salud

4 Médico, Grupo de Genética, INS

5 Médico en Servicio Social, Grupo de Genética, Instituto Nacional de Salud

6 Profesor, Departamento de Radiología. Universidad Nacional de Colombia

7 Bacterióloga, Grupo de Química Clínica, Instituto Nacional de Salud

8 Microbióloga, Grupo de Genética, Instituto Nacional de Salud

9 Antropólogo, Contratista, Instituto Nacional de Salud

10 Médico Reumatólogo, Profesor del Depto. de Reumatologia, Universidad Nacional de Colombia, Director Instituto Nacional de Salud
} 


\begin{abstract}
y los 64 casos que analizamos en el presente estudio, enfatizando nuestras observaciones, como el inicio temprano de las manifestaciones clínicas, ausencia de dolor o debilidad muscular, de alopecia, de niveles normales de fósforo y leve hiperparatiroidismo secundario, lo que indica una gran heterogeneidad en este tipo de raquitismo.
\end{abstract}

\section{INTRODUCCION}

A comienzos de 1991, con el objeto de solicitar ayuda al nivel central, se recibió en el Ministerio de Salud una lista con los nombres, fotografías y algunas placas de rayos- $X$, de un grupo de niños de origen afromestizo, de ambos sexos, que mostraban diferentes tipos y grados de deformidades de los miembros inferiores. Este primer grupo de niños provenía del corregimiento de La Toma, municipio de Suárez, al norte del departamento del Cauca.

En la lista de los nombres de estos niños, se observó que muchos de ellos compartían los mismos apellidos. En las fotografías se notó que a pesar de las deformidades óseas de los miembros inferiores, el aspecto general era bueno y en ninguno de ellos se notaban signos de desnutrición. La radiografías mostraban claramente signos de raquitismo

Considerando que no existiese un factor ambiental que pudiera causar este raquitismo, las observaciones anteriores permitían asumir que se podría tratar de una de las formas de raquitismo génito, autosómico recesivo, descritos en la literatura médica: los raquitismos dependientes de la vitamina D, tipos I y II (RDVD I y II) (1). La diferencia entre estas dos enfermedades radica en los niveles séricos de la $1 \alpha, 25(\mathrm{OH})_{2} \Delta_{3}$, el principal metabolito de la vitamina $D$.

Deficiencias de la enzima $25(\mathrm{OH}) \mathrm{D}_{3}-1 \alpha$ hidroxilasa resultan en niveles muy bajos o indetectables de la hormona $1 \alpha, 25(\mathrm{OH})_{2} \Delta_{3}$. Este defecto se clasifica como RDVD I (2). Los niveles muy altos de esta hormona son debidos a un defecto en la respuesta del órgano blanco, por déficit o insensibilidad del receptor y se clasifican como RDVD ॥ (3).

Los RDV tipos I y II, muestran cuadros clínicos, radiológicos y de laboratorio similares, con un rango muy amplio de severidad. Ambas enfer- medades tienen comienzo en la infancia o al inicio de la niñez, con la aparición del raquitismo, hipocalcemia, hipofosfatemia (en algunos) e hiperparatiroidismo secundario. En el tipo II, la mayoría de los pacientes presentan alopecia, que se han subclasificado como tipo II A y tipo IIB los que no la presentan (1).

La $1 \alpha, 25(\mathrm{OH})_{2} \Delta_{3}$, es una hormona esteroidea producida en los túbulos contorneados proximales del riñón, por la acción de la $25(\mathrm{OH}) \mathrm{D}_{3}-1 \alpha$ hidroxilasa, a partir del precursor, la $25(\mathrm{OH}) \mathrm{D}_{3}$ (4). Este paso está estrechamente regulado por las necesidades de calcio y fósforo en el organismo. Niveles bajos de estos elementos estimulan la glándula paratiroidea a secretar la paratohormona (PTH), la cual a su vez incrementa la producción de la enzima $25\left(\mathrm{OHD}_{3}-1 \alpha\right.$ hidroxilasa en los túbulos renales proximales (5).

Investigaciones de varios grupos han demostrado que la causa de la resistencia del órgano blanco en el RDVDII, es un defecto en el receptor de la vitamina D RVD presentel intracelularmente. (6-8).

Además de las células en donde se espera su acción más importante como son las del intestino delgado (células de borde en cepillo del duodeno), del riñón (túbulos contorneados proximales), del hueso (osteoblastos) y de la paratiroides, el RVD se ha observado en un abundante número de células de otros tejidos, en especial en los fibroblastos de la piel y en diferentes células del sistema inmune, como linfocitos y macrófagos activados y en varias líneas celulares provenientes de tejidos neoplásicos (9).

Se ha demostrado en los fibroblastos y otras células, que el catabolismo de la hormona se inicia con la formación del metabolito $24,25(\mathrm{OH})_{2} \mathrm{D}_{3}$ que se produce por acción la enzima $25(\mathrm{OH}) \mathrm{D}_{3}$ 24 hidroxilasa, que sólo es activada cuando el receptor está conservado. Por lo tanto, desde el punto de vista funcional, la demostración de la 
activación de esta enzima es una prueba indirecta de la presencia del receptor $(8,10)$.

Se estimlan de la misma manera, los linfocitos expresanelRVD cuando se estimulan con lectinas; este estímulo es inhibido por la hormona $1 \alpha, 25$ $(\mathrm{OH})_{2} \mathrm{D}_{3}$, por lo que no hay inhibición de los linfocitos estimulados de pacientes con defectos del RVD (11).

Las bases moleculares de este trastorno son debidas a diferentes tipos de anomalías funcionales del RVD. Estas caen dentro de tres categorías muy amplias fenotípicas: deficiencias de unión o ligamiento con el esteroide, anomalías de reconocimiento del DNA y defectos por transferencia o localización nuclear $(12,13)$. EI RVD humano es una proteína de aproximadamente $50 \mathrm{kD}$ que pertenece a la superfamilia de los genes de los receptores esteroide-tiroide-ácido retinoico (14). La proteína RVD, comprende dos dominios funcionales: un dominio de unión con la hormona esteroidea y un dominio de unión con el DNA (12-16).

EI DNA complementario que codifica el RVD ha sido clonado y secuenciado y se caracterizaron inicialmente 9 exones, de los cuales los exones 2 y 3 codifican el dominio que se une al DNA, mientras que los exones 7,8 y 9 codifican el dominio que se liga a la hormona vitamina $D$ $(16,17)$. Recientemente, se lograron estudiar varios clones genómicos que contenían el RVD y se determinó mejor la organización estructural de su gen. Se informó que éste contenía al menos 10 exones cuyos tamaños van desde aproximadamente 29 a 3.595 nucleótidos, los cuales junto con los intrones hacen que el gen alcance un tamaño entre 60 y 70 kilobases (18). Varias mutaciones puntuales en el gen del RVD han sido detectadas en pacientes con RDVD II, en las cuales un cambio en un aminoácido altera la estructura terciaria de esta proteína, tanto en el dominio que se liga al DNA, como en el dominio que se liga a la hormona (17-21). Sin embargo, en un paciente con este tipo de raquitismo no se encontró ninguna alteración en la secuencia de nucleótidos del cDNA del RVD (21).

Antes de que se realizaran estudios directos de gen, por medio del análisis del DNA, estudios bioquímicos con marcación radioactiva de la hormona tanto a nivel citoplasmático como nuclear, así como estudios del complejo receptor hormona por medio de gradientes de densidad de sacarosa, que muestran la presencia y funcionalidad del RVD, se describieron dos pacientes con RVD normal, pero que era incapaces de inducir la producción de 25-hidroxivitaminaD-24-hidroxilasa, por lo que se planteó la existencia de pacientes con RDVD II, con resistencia receptor-positivo (8-10)

\section{PACIENTES Y METODOS}

La mayor concentración de afectados viven en el corregimiento de La Toma, municipio de Suárez, al norte del departamento del Cauca, a 2 horas de Cali en automóvil. El corregimiento está localizado a 20 kilómetros de la cabecera municipal, tiene 5.117 habitantes, y en su mayoría descendientes híbridos, africano, índigena, español.

El origen de este asentamiento se remonta a los inicios de los años 30 , cuando esta tierra fue poseída de hecho, por los fundadores, un grupo de alrededor de 20 familias. Estas fueron familias de trabajadores, provenientes de la antigua hacienda Gelima, cercana a La Toma, en esa época perteneciente al municipio de Buenos Aires y hoy jurisdicción de Suárez (22). Las observaciones y estudios antropogenéticos serán objeto de una publicación posterior.

En todos los pacientes, la enfermedad se inicia con un aumento del perímetro en la diáfisis distal del fémur y proximal de la tibia de uno o ambos miembros inferiores, referido por los familiares como un "engrosamiento de las rodillas", seguido por la aparición de las deformidades en miembros inferiores, poco después de empezar la deambulación. Algunos refieren dolor en las rodillas, el cual en ningún caso ha sido invalidante. Todos realizan sus actividades normales incluso las prácticas deportivas escolares.

Sesenta y cuatro de los pacientes, más severamente afectados, de ambos sexos $(40,6 \%$ hombres y $59,4 \%$ mujeres) y con una edad promedio de 9,1 años (rango 2,28) fueron estudiados desde el punto de vista clínico, radiológico y de laboratorio. A todos se les realizó una detallada historia clínica, que ha sido revisada periódicamente por uno de los autores (WP) (figura 1.) 
A.GIRALDO, W. PINO, M.PINEDA Y COLABORADORES
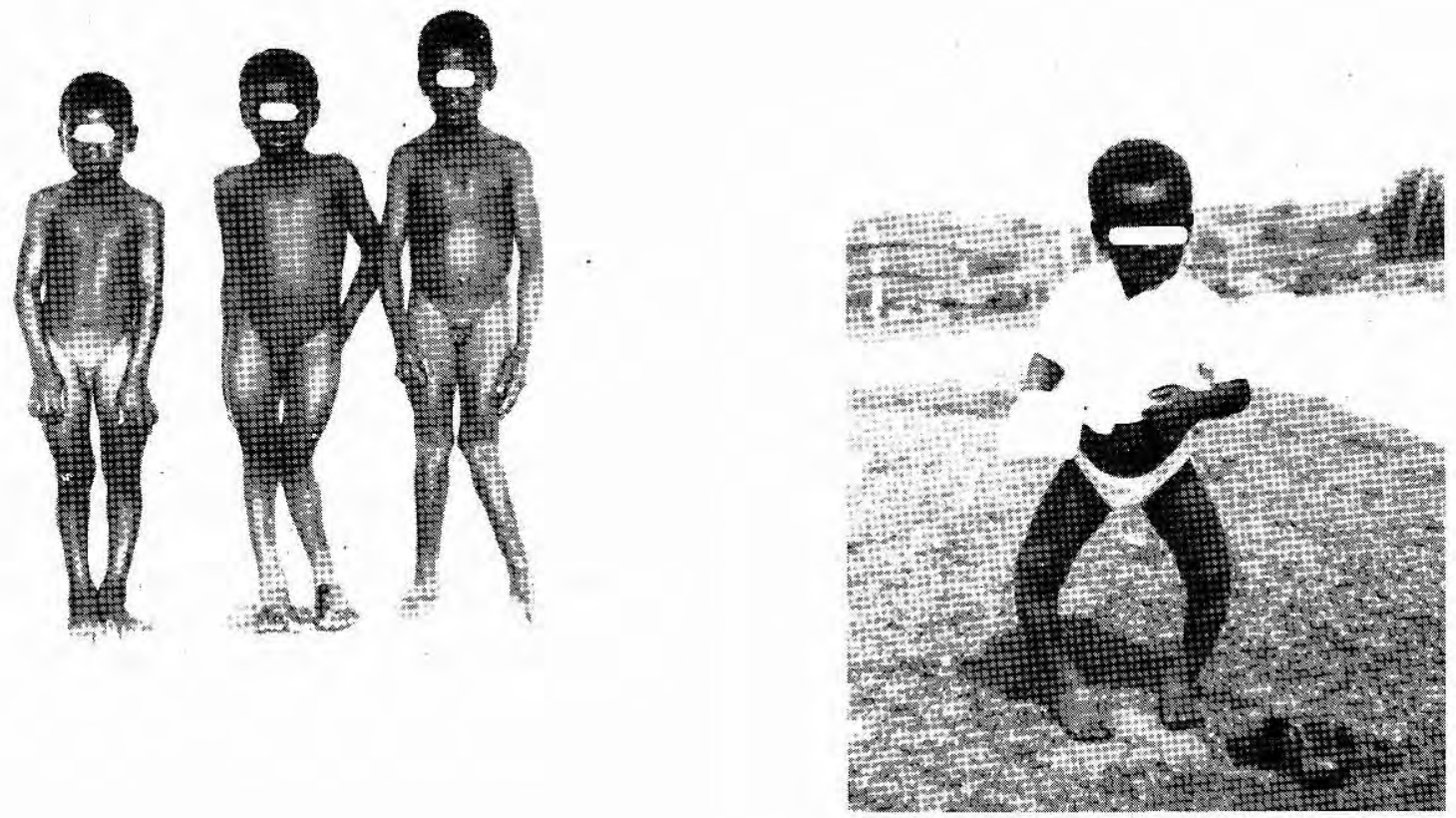

Figura 1. Pacientes con severa deformidad en miembros inferiores procedentes del corregimiento de La Toma.
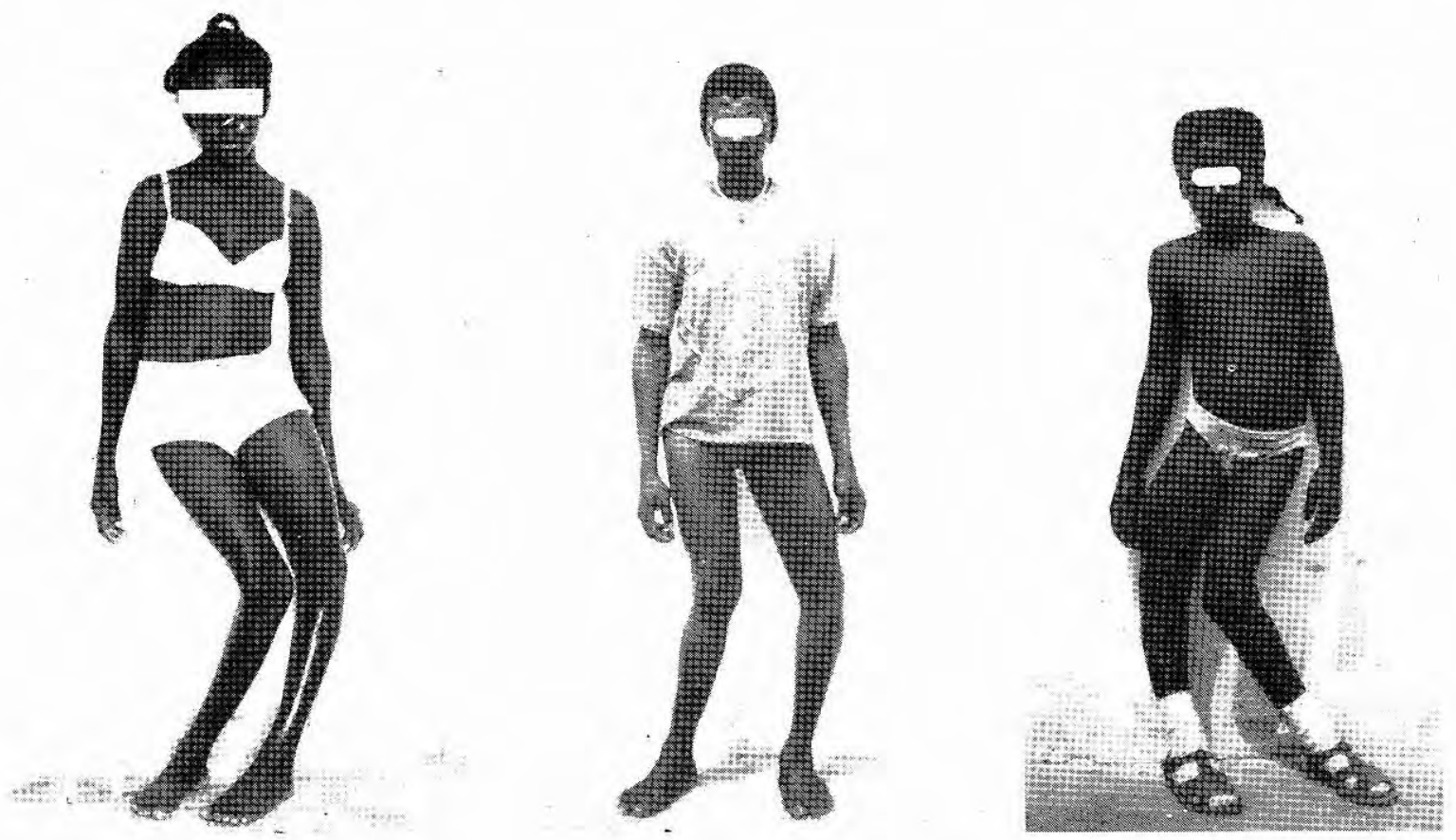
Se realizaron las siguientes pruebas de laboratorio con reactivos comerciales en el Grupo de Química Clínica del INS: calcio y fósforo séricos, según el método colorimétrico de punto final (Labtest); la medición de la fosfatasa alcalina se realizó por la técnica cinética de dos puntos (Monotest). Para la determinación de las proteínas totales, se siguió el método de Biuret (Miles) y para la albúmina, el método colorimétrico de punto final (Miles).

La medición de la $25(\mathrm{OH}) \mathrm{D}_{3}$ y de la $1,25(\mathrm{OH})_{2} \mathrm{D}_{3}$ en los primeros 8 , pacientes se realizó por el método de Reinhart (23), en el Instituto de Bioquímica de Glasgow, Gran Bretaña. (Dr. Graham $H$. Beastall). Otros 30 pacientes fueron estudiados por el mismo método en el laboratorio de metabolismo mineral del Centro Médico del Suroeste de la Universidad de Dallas, E.U.A. (Dr. Joseph Zerwekh y Maritza Pineda). En este mismo centrosedeterminólaactividad delaenzima25(OH)D324 hidroxilasa, por el método de cromatografía líquida de alta eficiencia (HPLC) en fase reversa (8). La hormona paratiroidea se determinó por el método de análisis radioinmunométrico de dos sitios (Allegro), en la Unidad Endocrina del Hospital General de Massachussetts deBoston, E.U.A. (Dr. HaraldJueppner y Dr. David Freyle). Otro grupo de pacientes fue estudiado por el mismo método, pero con reactivos no comerciales en el laboratorio de metabolismo mineral, antes mencionado, en Dallas.

También, se hicieron determinaciones en orina de 24 horas, de calcio y fósforo y de aminoácidos en11pacientes; estos últimos se hicieron cualitativamente en pruebas de tubo de ensayo (24) y semicuantitativamente por cromatografía de capa fina (25) (Grupo de Genética, INS). En otro grupo de 10 pacientes, se determinan los valores de calcio y fósforo en orina de 24 horas después del tratamiento y las medias de estos elementos se compararon por medio de la t de Student (26).

A 22 pacientes se les practicaron estudios radiológicos de miembros superiores, inferiores y cráneo en el Hospital de Santander de Quilichao, Cauca, los cuales fueron revisados detalladamente por uno de los autores (FN).

A un subgrupo de los pacientes se les determinaron con reactivos comerciales varios grupos sanguíneos, $A B O, R h(C c D E e)$, MNSs, Duffy (Fya, $\mathrm{Fyb}, \mathrm{Fy})$, Kell (K, k) y Xg (Biotest) y hemoglobinas (27). (Grupo de Genética del INS). A un grupo de familias con hijos afectados y sanos se les determinaron los antígenos del sistema mayor de histocompatibilidad, de clase I y clase II (Laboratorio de Genética Universidad Nacional), cuyos resultados serán publicados en otro informe.

A dos de los pacientes más severamente afectados, se les tomó una biopsia de piel, para realizar cultivos de fibroblastos y estudios moleculares del mRNA y del cDNA del receptor. Estos se practicaron en el Instituto de Genética Molecular de la Escuela de Medicina de la Universidad de Baylor, Houston, E.U.A, (Dr. Mark Hughes y Dr. Luis Fernando García). Un informe detallado de los hallazgos observados en estos estudios se hará por aparte.

Una muestra de 109 parientes en primer grado, sanos, de ambos sexos y con una edad promedio de 28,9 años (rango 2,68), también fue estudiada y las medias de los valores observados fueron comparados estadísticamente por medio de la t de Student (26).

Aunque se tenían razones suficientes para no sospechar la existencia de factores externos, se decidió descartar la presencia de contaminantes ambientales, como fitatos, que pudieran impedir una absorción normal del calcio a nivel intestinal $\mathrm{u}$ otras sustancias que pudieran tener alguna relación con la enfermedad (Laboratorio de Sanidad del Ambiente, INS)

Una vez establecido el diagnóstico y clasificada la enfermedad, se inició el tratamiento según el protocolo de Marx (28) modificado. Se partió de la base de que los pacientes necesitaban más calcio y que se podría lograr incrementar su paso a las células del intestino, sobresaturándolos con calcio y hormona vitamina $\mathrm{D}\left(1 \alpha, 25(\mathrm{OH})_{2} \mathrm{D}_{3}\right.$ Un preparado comercial para uso veterinario fue el único en el comercio que contenía las cantidades apropiadas de calcio, por lo que se solicitó al productor que hiciera una etiqueta especial para nuestros pacientes. Este preparado se suministró en dos dosis diarias, para una ingesta total de $1.500 \mathrm{mg} /$ día de calcio y $1424 \mathrm{mg} /$ día de fósforo. También, se les suministró ug/día 0,50 mcrg/día de una presentación comercial de la hormona vitamina $D$, en dos dosis. 
Después de un año de tratamiento se hizo una comparación estadística entre los valores de laboratorio pre y post tratamiento, según la prueba de $t$ de Student apareada (26).

\section{RESULTADOS}

El desarrollo psicomotor es normal en la mayoría de los pacientes, algunos padres han informado un inicio tardío de la deambulación. El examen físico de todos los pacientes mostró un desarrollo adecuado para las edades correspondientes, excepto por las deformidades en miembros inferiores, que hacen que la talla en éstos sea más baja. Un paciente presenta retardo mental leve, con convulsiones ocasionales, al parecer secundario a trauma obstétrico. Ningún paciente ha presentado tetania ni alopecia.

El $50 \%$ de los pacientes presentaba deformidad de las rodillas en varo, el $42,2 \%$ deformidad en valgo y un $7,8 \%$ deformaciones mixtas.

Los estudios radiológicos de los miembros inferiores de los diferentes pacientes, mostraron una marcada incurvación de la porción distal de la diáfisis femoral y de la porción proximal de la diáfisis tibial. Se observó un ensanchamiento de las metáfisis y una disminución generalizada de la densidad ósea. La cortical se encontró muy delgada así como también se observó un incremento de las trabéculas medulares y numerosas líneas de Park. Se resalta el hecho de que la epífisis y los espacios articulares se encuentran poco afectados. No se observó en ninguno de los estudiados, compromiso de los miembros superiores, cráneo o reja costal (rosario raquítico), (figura 2.)

El 40,6\% de los pacientes tenían valores de calcio por debajo de los valores normales $(<8.8$ $\mathrm{mg} / \mathrm{dl}$ ), el $44 \%$ de los pacientes valores de calcio sérico dentro límites normales y el $15,6 \%$ valores por encima de lo normal (>11,0 mg/dl). Para esos mismos rangos, los controles sanos, parientes en primer grado de los pacientes, fueron $18,3 \%$ $(<8,8 \mathrm{mg} / \mathrm{dl}), 53,2 \%$ dentro de los limites normales y $28,5 \%(>11,0 \mathrm{mg} / \mathrm{dl})$, pero, al comparar la media de los enfermos con la media de los controles sanos, la diferenciafue significativamente menor en los pacientes (tabla 1).
La media de los valores del fósforo sérico a su vez, fue significativamente mayor en los afectados que en los controles sanos, no encontrándose ninguno de los pacientes con valores por debajo del rango normal (<2,5 mg/dl), (tabla 1$)$.

La fosfatasa alcalina se observó marcadamente incrementada en los afectados, sólo el $3 \%$ de ellos mostraron valores dentro de lo normal (98-279 U/L). El 51,4\% de los controles sanos, parientes en primer grado, mostraron valores de la enzima dentro del rango normal, mientras que el $48,6 \%$ restante mostraron valores altos de la enzima, algunos de ellos sustancialmente por encima de los valores normales convencionales. Sin embargo, la diferencia entre afectados y controles sanos fue altamente significativa (tabla 1).

Por su parte, los valores de las proteínas totales mostraron diferencias significativas siendo la media ligeramente más alta en los sanos, pero la albúmina no mostró diferencias estadísticamente significativas entre los afectados y los controles sanos, (tabla 1).

Los valores de la $25(\mathrm{OH}) \mathrm{D}_{3}$ se encontraron significativamente más bajos en los pacientes que en los controles, pero ampliamente dentro de lo normal. Por el contrario, los valores de la hormona $1,25(\mathrm{OH})_{2} \mathrm{D}_{3}$ se encontraron marcadamente elevados en lo pacientes en comparación con los controles, diferencia muy significativa, aunque estos últimos mostraron valores ligeramente por encima de lo normal (tabla 2). Este resultado es el determinante para el diagnóstico y para clasificar el tipo de raquitismo, pues la hormona alta significa falla en el receptor, por lo tanto RVDD tipo II. Por no presentar alopecia, nuestros pacientes corresponden al tipo IIB, según McKusick (1).

La paratohormona se encontró en los límites normales altos en la mayoría de los pacientes, pero en algunos de éstos estuvo muy por encima del rango normal por lo que la media fue significativamente más alta en los pacientes que en los controles (tabla 2).

La media del calcio y del fósforo en orina de 24 horas, se encontró en un grupo de 11 pacientes, muy por debajo de los valores normales (tabla 3). 
A

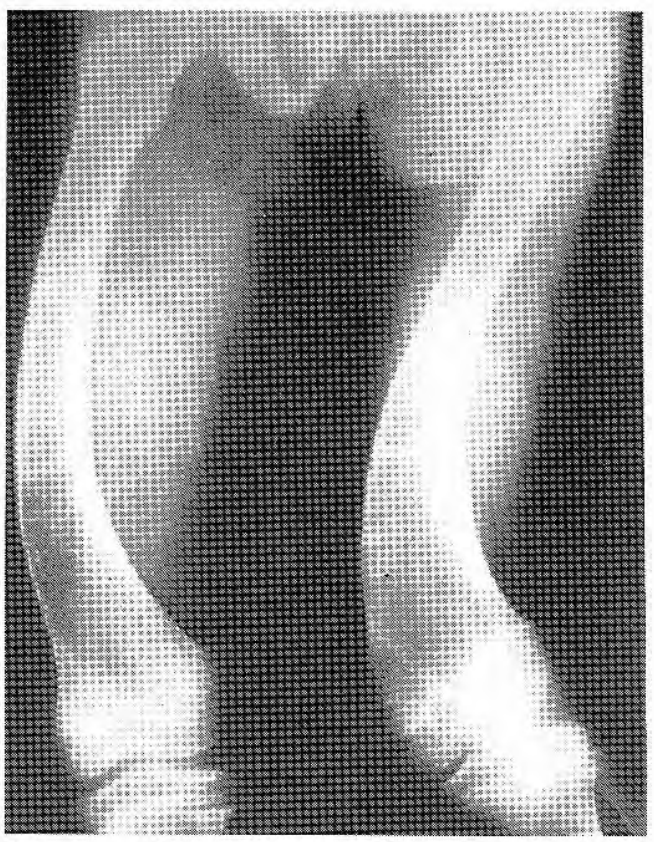

C

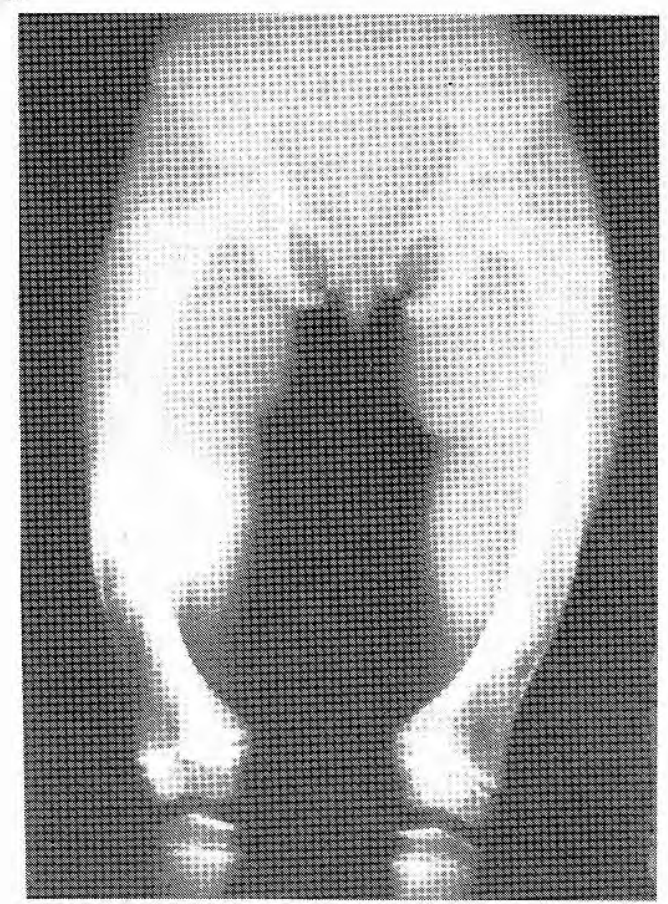

B

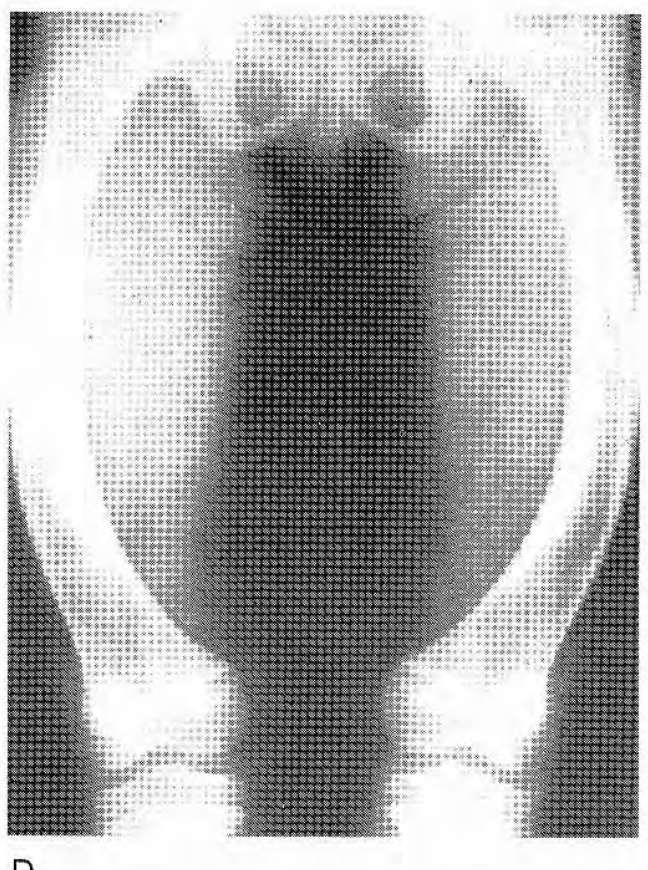

D

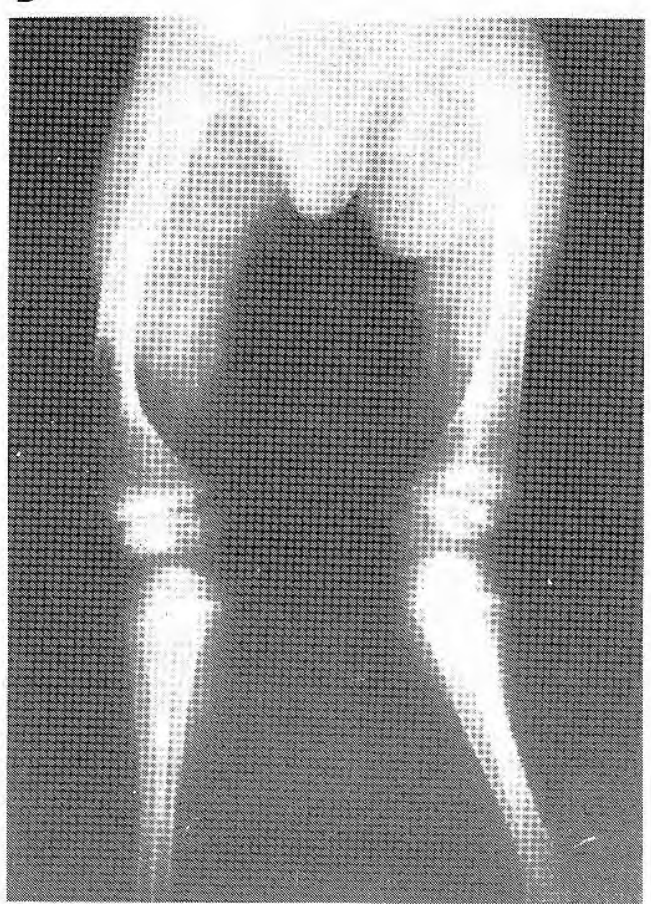

Figura 2: A, marcada deformidad de ambos fémures, con ensanchamiento diafisiario distal, disminución de la cortical y osteopenia. B. severa deformación en varo de los fémures con ensanchamiento distal de éstos, con adelgazamiento cortical y ensanchamiento metafisiario. C. Angulación en varo de los fémures, con ensanchamiento diafisiario, más marcado en los tercios distales: coxa vara bilateral: en los extremos distales de los fémures es notorio el adelgazamiento cortical con resorción endóstica. D. severa deformación en varo de ambos fémures. con resorción ósea generalizada (epifisis, diáfisis y metáfisis) con ensanchamiento diafisiario y metafisiario distal del femur y proximal de huesos de la pierna: se observa además impresión epifisiaria sobre la fisis o placa de crecimiento 
TABLA 1.Análisis de laboratorio; comparación de los valores promedios .

\begin{tabular}{|c|c|c|c|c|c|}
\hline & \multicolumn{2}{|c|}{ NORMALES } & \multicolumn{2}{|c|}{ AFECTADOS } & \multirow[b]{2}{*}{$\mathrm{p}$} \\
\hline & Media & D.S & Media & D.S. & \\
\hline $\begin{array}{l}\text { CA sérico } \\
(\mathrm{VN}: 8,8-11,0 \mathrm{mg} / \mathrm{dl})\end{array}$ & 10,24 & 1,74 & 9,25 & 1,52 & 0,00046 \\
\hline $\begin{array}{l}\text { P sérico } \\
(\mathrm{VN}: 2,5-4,8 \mathrm{mg} / \mathrm{dl})\end{array}$ & 3,89 & 0,99 & 4,58 & 0,96 & 0,00009 \\
\hline $\begin{array}{l}\text { Fosfatasa alcalina } \\
\text { (VN: } 98-279 \mathrm{U} / \mathrm{l})\end{array}$ & 473,0 & 473,3 & 1404,1 & 1092,2 & 0,0000001 \\
\hline $\begin{array}{l}\text { Proteínas totales } \\
\text { (VN: } 6-8 \mathrm{~g} / \mathrm{dl})\end{array}$ & 7,71 & 0,73 & 7,33 & 0,83 & 0,002469 \\
\hline $\begin{array}{l}\text { Albúmina } \\
\text { VN: } 3,5-5,0 \mathrm{~g} / \mathrm{dl}\end{array}$ & 4,24 & 0,49 & 4,26 & 0,62 & 0,830329 \\
\hline
\end{tabular}

Normales: $n=109$.

Afectados: $n=64$

TABLA 2. Comparación de los promedios de los metabolitos de la vitamina $\mathrm{D}$ y paratohormona

\begin{tabular}{|c|c|c|c|c|c|}
\hline & \multicolumn{2}{|c|}{ NORMALES } & \multicolumn{2}{|c|}{ AFECTADOS } & \multirow[b]{2}{*}{$P$} \\
\hline & Media & D.S & Media & D.S. & \\
\hline $\begin{array}{l}25(\mathrm{OH}) \mathrm{D3} \\
(\mathrm{VN}: 15-100 \mathrm{nmol} / \mathrm{l})\end{array}$ & 76,0 & 9,3 & 43,2 & 15,0 & 0,000262 \\
\hline $\begin{array}{l}1,25(\mathrm{OH}) 2 \mathrm{D} 3 \\
(\mathrm{VN}: 20-120 \mathrm{pmol} / \mathrm{l})\end{array}$ & 120,1 & 43,0 & 322,4 & 105,8 & 0,00036 \\
\hline $\begin{array}{l}\text { PTH } \\
(\mathrm{VN}: 10-65 \mathrm{pg} / \mathrm{ml})\end{array}$ & 17,2 & 11,2 & 69,7 & 144,2 & 0,001592 \\
\hline
\end{tabular}

Vitamina D3: Normales: $\mathrm{n}=8$. Afectados: $\mathrm{n}=8$

Paratohormona Normales: $n=83$. Afectados: $n=56$ 
TABLA 3. Análisis de calcio y fósforo en orina de 24 horas

\begin{tabular}{lcc}
\hline & Media & D.S. \\
\hline & & \\
CALCIO & 4,9 & 3,3 \\
(VN: $60-180 \mathrm{mg})$ & & \\
FOSFORO & 194,7 \\
(VN: $340-1000 \mathrm{mg})$ & & \\
AMINOACIDOS & Negativo. \\
\hline
\end{tabular}

$n=11$

No se encontró aminoaciduria en ninguno de los pacientes estudiados (tabla 3). Tampoco se encontraron trazas de fitatos ni de ningún otro contaminante ambiental, que pudiera ser la causa de malabsorción de calcio en estos pacientes.

Los estudios moleculares del mRNA y el cDNA realizados en el Instituto de Genética Molecular de la Escuela de Medicina de Baylor, la secuenciación de nucleótidos del gen del receptor para la vitamina $D$ (RVD) fue normal. Sin embargo, el estudio en los mismos fibroblastos de los cuales se extrajo el mRNA $y$ posteriormente el CDNA, de la enzima 25(OH)D3-24. hidroxilasa, mostró una muy baja inducción de ésta.

En un grupo de 21 pacientes, se analizaron los datos de laboratorio en suero, pre y post tratamiento un año después, y todos los valores tendieron normalizarse, incluyendo el fósforo que se encontraba en los afectados ligeramente más alto que en los controles (tabla 4).

TABLA 4. La comparación de los promedios promedios de química snguínea en pacientes pre y post-tramiento

\begin{tabular}{|c|c|c|c|c|c|}
\hline & \multicolumn{2}{|c|}{ PRE-TRATAMIENTO } & \multicolumn{2}{|c|}{ POST-TRATAMIENTO } & \multirow{2}{*}{$\begin{array}{r}\text { INTERVALOS } \\
\text { DE CONFIANZA }\end{array}$} \\
\hline & Media & D.S & Media & D.S & \\
\hline $\begin{array}{l}\text { CA SERICO } \\
(\mathrm{VN}: 8.8-11.0 \mathrm{mg} / \mathrm{dl})\end{array}$ & 7,92 & 0,88 & 8,74 & 0,87 & $(0.59-1.43)$ \\
\hline $\begin{array}{l}\text { P SERICO } \\
(\mathrm{VN}: 2.5-7.0 \mathrm{mg} / \mathrm{dl})\end{array}$ & 4,88 & 0,94 & 4,60 & 0,84 & $(0.29-0.97)$ \\
\hline $\begin{array}{l}\text { FOSF.ALCALINA } \\
(\mathrm{VN}: 98-279 \mathrm{U} / \mathrm{l})\end{array}$ & 1559 & 761,4 & 1004 & 417,3 & $(252,4-884,9)$ \\
\hline $\begin{array}{l}\text { PROT. TOTALES } \\
(\text { VN: } 6.0-8.7 \mathrm{~g} / \mathrm{dl})\end{array}$ & 7.43 & 0.75 & 7,01 & 0,63 & $(0,45-1,07)$ \\
\hline $\begin{array}{l}\text { ALBUMINA } \\
(\text { VN: } 3.0-5.4 \mathrm{~g} / \mathrm{dl})\end{array}$ & 4.37 & 0.55 & 4,27 & 0,47 & $(0,33-0,79)$ \\
\hline
\end{tabular}

Pre-tratamiento: $n=21$.

Grados de libertad $=20$

Postratamiento: $n=21$.

$u=99$ 
En la orina de 24 horas de un grupo de pacientes (diferente al que se estudió inicialmente para orina de 24 horas), se observó inicialmente franca y significativa mejoría, valores de calcio y fósforo al compararla con los! valores del grupo inicial de pacientes, aunque siguen estando por debajo de lo normal (tabla 5).

Muchos de los pacientes y sus familiares aseguran también una mejoría clínica en aquéllos cuyas deformidades óseas no eran tan graves.

En la zona del corregimiento de La Toma donde se concentran la mayoría de los pacientes estudiados, son muy frecuentes los apellidos: Lucumí, Carabalí y González, entre otros; lo cual sugiere indirectamente una alta frecuencia de consanguinidad. Se levantaron numerosos árboles genealógicos (CA) que muestran consanguinidad próxima y remota cuyo análisis detallado se publicará separadamente (figura 3 ). Estos hallazgos corroboran la herencia autosómica recesiva de este tipo de raquitismo.

TABLA 5. Comparación de los promedios de calcioy fósforo en orina de $\mathbf{2 4}$ horas en pacientes pre y post-tratamiento

PRE-TTO D.S POST-TTO D.S.

\begin{tabular}{lcccc} 
CALCIO & 4,9 & 3,3 & 12,6 & 9,8 \\
\hline (VN:60-180 mg) & & & & \\
FOSFORO & 193,3 & 194,7 & 325,7 & 258,3 \\
(VN:340-1000 mg) & & & & \\
AMINOACIDOS & Negativo & Negativo. \\
\hline
\end{tabular}

Pre-tratamiento: $n=11$

Postratamiento: $n=10$
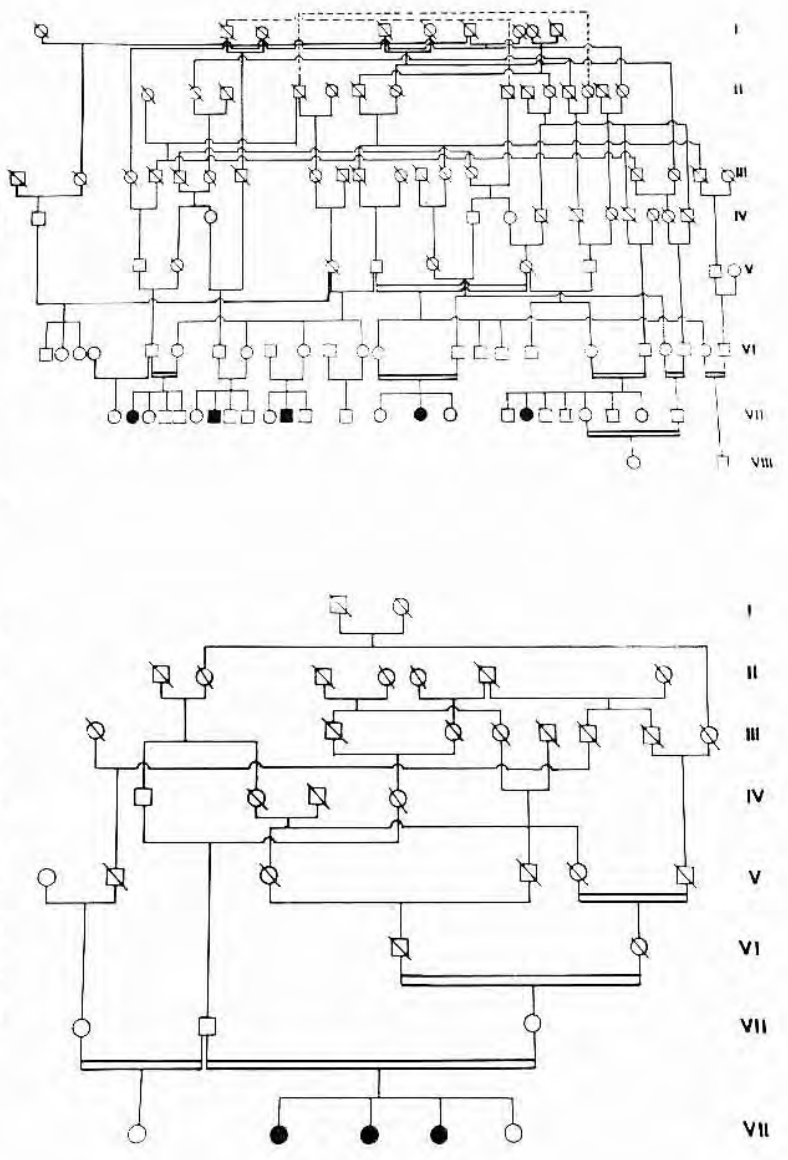

Figura 3: Arboles genealógicos de pacientes del corregimiento de La Toma que muestran consanguinidad próxima y remota.

\section{DISCUSION}

El raquitismo fue un serio problema de salud en una proporción apreciable de niños y jóvenes a finales del siglo pasado en Norteamérica y Europa, hasta cuando se descubrió que se podía curar con aceite de hígado de bacalao. Posteriormente, se demostró que el factor curativo era la nueva vitamina $D$, así como también la participación de la luz ultravioleta en su metabolismo. Muy pronto se llegó a su síntesis química y a su producción en grandes cantidades, lo que permitió en la práctica que se erradicara el raquitismo de esos países, antes de los años $40(5,28)$. En los países subdesarrollados aún se observan casos de raquitismo en grupos con alta prevalencia de desnutrición (29). En Colombia, el raquitis- 
mo carencial es cada vez menos frecuente, los pediatras y médicos en retiro lo recuerdan, en especial en las zonas rurales y al parecer es de observación muy ocasional en la actualidad.

Al iniciarse los tratamientos masivos con vitamina $D$, se empezaron a describir algunos tipos de raquitismos resistentes al tratamiento y a dilucidarse los mecanismos de acción de esta vitamina y sus diferentes metabolitos. De éstos se han estudiado especialmente la $1\left(1,25(\mathrm{OH})_{2} \mathrm{D}_{3}\right.$ que se detectó como el más activo de los metabolitos y verdadera hormona esteroidea $(4,5)$ y su precursor la $25(\mathrm{OH}) \mathrm{D}_{3}$ que a su vez necesita de la acción de la enzima $25(\mathrm{OH}) \mathrm{D}_{3}-10$ hidroxilasa, para su transformación en la hormona mencionada $(2,4)$.

En 1978, Brooks describió en un caso esporádico, el llamado raquitismo dependiente de vitamina D tipo II, en el cual el defecto se identifica por niveles muy altos de la hormona lo que se atribuyó a falta de funcionamiento del receptor (3).
Se han descrito en la literatura 20 casos, varios de ellos esporádicos y otros familiares, con esta forma de raquitismo $(3,17,30-40)$, es decir en todos ellos se ha encontrado una elevación de la $1 u, 25(\mathrm{OH})_{2} \mathrm{D}_{3}$ (tabla 6). Como se ha mencionado, el RDVD tipoll ha sido subclasificado, de acuerdo con la presencia o no de alopecia, en IIA y IIB respectivamente (1). Cinco de los 20 pacientes de la tabla no muestran alopecia, por lo que se subclasificarian como tipo IIB, al igual que nuestros pacientes (tabla 6).

Sin embargo, entre los pacientes sin alopecia descritos (tipo IIB, tabla 6) y nuestros pacientes (tabla 7), existen importantes diferencias clínicas y metabólicas. En tres de aquéllos se encontró aminoaciduria $(3,30)$, la cual no se observó en ninguno de los 11 pacientes de nuestro grupo a quienes se les investigó (tabla 3). En dos de los pacientes tipo IIB, los síntomas y signos del raquitismo se iniciaron entre los doce y los quince años, en los otros tres se inició la enfermedad, al igual que en nuestros pacientes, en los primeros años de vida (tabla 7).

TABLA 6. Aspectos clinicos y de laboratorio de los casos con RVDD TIPOS IIA Y II, publicados en la literatura

\begin{tabular}{lcccccc}
\hline Tipo IIA & \multicolumn{2}{c}{ TOTAL POSITIVOS } & & & & \\
& POS & NEG & POS & NEG & CASOS & $\%$ \\
\hline & & & & & & \\
Deformidades óseas & 14 & 1 & 4 & 1 & 18 & 93,3 \\
Alopecia & 15 & 0 & 0 & 5 & 15 & 75,0 \\
Inicio temprano & 15 & 0 & 3 & 2 & 18 & 90,0 \\
Dolor/debilidad & 3 & 12 & 4 & 1 & 7 & 35,0 \\
Consanguinidad & 13 & 2 & 0 & 5 & 13 & 86,6 \\
Hiper 1,25(OH)2D3 & 15 & 0 & 5 & 0 & 20 & 100,0 \\
Hiper fosfatasa alcalina & 15 & 0 & 5 & 0 & 15 & 100,0 \\
Hiper paratohormona. & 14 & 1 & 5 & 0 & 19 & 95,0 \\
Hipocalcemia & 12 & 3 & 5 & 0 & 17 & 85,0 \\
Hipofosfatemia & 9 & 6 & 2 & 3 & 11 & 55,0 \\
Aminoaciduria & 5 & 10 & 3 & 2 & 8 & 40,0 \\
\hline Total Pacientes Publicados & 15 & 5 & 20 & & 20 & \\
\hline
\end{tabular}


TABLA 7. Aspectos clínicos y de laboratorio de los casos con RVDD tipo en el presente estudio

\begin{tabular}{lcccc}
\hline & POS & NEG & $\begin{array}{c}\text { TOTAL CASOS } \\
\text { ESTUDIADOS }\end{array}$ & $\begin{array}{c}\text { TOTAL } \\
\text { POSITIVOS \% }\end{array}$ \\
\hline Deformidades óseas & 64 & 0 & 64 & 100.0 \\
Alopecia & 0 & 64 & 64 & 0.0 \\
Inicio Temprano & 50 & 14 & 64 & 78.5 \\
Dolor/Debilidad & 4 & 50 & 64 & 21.8 \\
Consanguinidad & 42 & 22 & 64 & 65.6 \\
Hiper 1,25(OH)2D3 & 8 & 0 & 8 & 100.0 \\
Hiper Fosfat.Alcalina & 64 & 0 & 64 & 100.0 \\
Hiper PTH & 7 & 49 & 56 & 12.5 \\
Hipocalcemia & 35 & 29 & 64 & 54.6 \\
Hipofosfatemia & 0 & 64 & 64 & 0.0 \\
Aminoaciduria & 0 & 11 & 11 & 0.0 \\
\hline
\end{tabular}

El calcio en todos los pacientes tipo IIB descritos (tabla 6), se encontró por debajo de los niveles normales; en nuestros pacientes, se encontró inferior a lo normal en el $40.6 \%$ (tabla 7). El fósforo fue norma; l en tres de esos (tabla 6) pacientes y bajo en dos de ellos; el fósforo en nuestros pacientes fue normal, en los rangos superiores.

En todos los pacientes tipo IIB (tabla 6) se encontró hiperparatiroidismo secundario. En nuestros pacientes, 7 de los 56 estudiados para la PTH (12.5\%), mostraron valores por encima de lo normal, pero la mayoría se encontraron en los límites superiores normales (tabla 7).

Con respecto a los pacientes con alopecia o tipo IIA (tabla 6), en todos se observó un cuadro clínico más severo, todos tuvieron inicio temprano; doce de los quince pacientes tipo IIA presentaron hipocalcemia y en tres de ellos el calcio fue normal $(5,40)$. En 9 de los 15 se observó hipofosfatemia, en 5 pacientes ei fósforo se encontró normal y en uno se observó alto (33). En 14 de estos 15 pacientes se encontró hiperparatiroidismo secundario, pero en uno de ellos la PTH fue normal (40).
Ninguno de los pacientes informados como tipo IIB (tabla 6) presentó antecedentes de consanguinidad. De los 15 pacientes tipo IIA (tabla 6) 13 presentaron antecedentes de consanguinidad. En el grupo de nuestros pacientes, a 42 de los $64(65.6 \%)$, se les detectaron antecedentes de consanguinidad (tabla 7).

En todos los casos descritos y en los pacientes de este estudio se observó una marcada elevación de la fosfatasa, alcalina, que aunque inespecífico, en este raquitismo indica actividad osteoblástica incrementada $(5,2,28)$.

Según estas comparaciones, tenemos elementos que indican una gran heterogeneidad en el RDVD II, tanto genética como eventualmente no genética. Esto quiere decir que en algunos de los pacientes descritos, en particular los casos esporádicos (aunque no necesariamente por eso), el mecanismo por el cual se haya presentado la enfermedad podría no tener una etiología genética.

Esta gran heterogeneidad se reflejaría en la gran diversidad clínica de todos los pacientes descritos incluyendo nuestros pacientes. Otro aspecto muy importante que se observa en los pacientes descritos, pero, en particular dentro del 
grupo de nuestros pacientes, es la expresividad variable o diferencias en las manifestaciones clínicas de un mismo trastorno genético, entre diferentes familias o intrafamiliarmente.

Esta variación en la expresión es debida en parte al trasfondo genético, que es diferente en cada individuo y se manifiesta tanto desde el punto de vista clínico como en los valores del laboratorio. Esto explica la mayoría de las diferencias observadas dentro del grupo de nuestros pacientes y los descritos en la literatura.

En este estudio estamos analizando los hallazgos clínicos y de laboratorio de 64 pacientes y comparándolos con los 20 casos descritos en la literatura. Es importante resaltar el hecho de que pocas veces se tiene la oportunidad de estudiar un número tan grande de individuos afectados con una enfermedad genética rara, en este caso autosómica recesiva, en una zona geográfica circunscrita, de manera semejante a lo observado en la población Amish de los Estados Unidos (42) o a las poblaciones francocanadienses en las cuales se han estudiado varias enfermedades genéticas, incluso el RVDD Tipo I (45). A este fenómeno de acumulación de pacientes en un área se le denomina foco ("cluster"), y se debe al denominado efecto fundador, consecuencia de la deriva genética.

La deriva genética es la fluctuación al azar de la frecuencia de los genes en poblaciones pequeñas. Cuando un gen, por baja que sea su frecuencia en una población inicial, debido al azar, está presente en uno o varios individuos de una familia o grupo que migra y constituye una nueva población, la frecuencia de ese gen en esta nueva población y en su siguiente generación puede ser mucho mayor, porque el grupo migrante no representa la frecuencia del gen de la población original. Esta situación es más posible cuando la nueva población está aislada o semiaislada. Cuando la frecuencia del gen raro se fija en la nueva población con una frecuencia alta, como resultado de los circunstancias anteriores se dice que se ha dado un efecto fundador.

Otro aspecto preponderante que resulta de estudiar un número grande de pacientes y de familiares sanos, es el poder hacer comparaciones estadísticas entre los dos grupos. En este estudio las diferencias significativas al 5 y al $1 \%$, fue posible detectarlas comparando las medias de todas las variables de laboratorio.

En algunos de los casos publicados en la literatura (17-21) se encontraron mutaciones puntuales en el gen del RVD que explica su hipofuncionalidad debida a defectos estructurales del RVD.

El gen del receptor para la vitamina $D$ de nuestros pacientes, al igual que otros previamente publicados $(8,10,21)$, no mostró defectos en la secuenciación de nucleótidos por el estudio del cDNA, lo que hace suponer una estructura proteica normal. Sin embargo por encontrarse tan alta la $1 \alpha, 25(\mathrm{OH})_{2} \Delta_{3}$ se evidencia un trastorno en su funcionamiento que puede ser debido a un defecto en su afinidad o capacidad, lo que se explica por la incompetencia en iniciar el catabolismo de la hormona al no inducir la actividad de la 25(OH)D3-24 hidroxilasa.

Por lo anterior, consideramos que la alopecia no sería un elemento importante en la subclasificación en los tipos IIA y IIB ya que puede ser simplemente una manifestación clínica de las formas más severas de raquitismo tipo Il; y así como éste se clasificó por tener niveles incrementados de la $1 \alpha, 25(\mathrm{OH})_{2} \mathrm{D}_{3}$, la secuenciación normal o anormal del gen del RVD podría más bien subclasificar la enfermedad según se tengan o no mutaciones en el gen del receptor. En estos últimos el defecto posiblemente se encuentre a nivel postranscripcional o en genes que produzcan otras proteínas estructurales o enzimas que intervengan en los mecanismos de acción de RVD.

En los casos publicados de RVDD tipo II se observa una gran variedad en cuanto al tipo de tratamiento suministrado a los pacientes y a la respuesta que se ha obtenido. En ninguno de ellos se ha observado respuesta a la $1,25(\mathrm{OH})_{2} \mathrm{D}_{3}$ administrada en dosis convencionales, algunos han respondido a dosis muy altas de este metabolito (30) y en otros casos descritos se ha obtenido mejoría utilizando: $25(\mathrm{OH})$ D3 (32), vitamina D2 (40), vitamina D3 (3), fósforo únicamente (31), calcio endovenoso en infusiones nocturnas (43) y aún con 24,25(OH)2 D3 (13), considerado como el metabolito que inicia el catabolismo 
de la forma hormonal (44). En nuestros pacientes hemos obtenido respuesta favorable utilizando el doble de la dosis convencional de 1,25 $(\mathrm{OH}) 2$ D3 pero que han sido administradas en combinación con calcio y fósforo.

El tratamiento que se planeó para nuestros pacientes se basó en lo propuesto por Marx (28), con algunas modificaciones (46), que se hicieron bajo el presupuesto de que se debía usar una dosis alta de $1 \alpha, 25(\mathrm{OH})_{2} \Delta_{3}$, aunque no necesariamente y tan alta como la propuesta por Marx, puesto que nuestros pacientes tienen endógenamente la hormona muy alta. Por otra parte, el calcio se suministró en una misma presentación con el fósforo, debido a que se encontró esta preparación comercial a un costo razonable y aunque el fósforo en nuestros pacientes se encontraba incluso un poco más alto que en los controles, se consideró que podría ser favorable como sustrato para la mejor mineralización ósea.

Los cambios en los datos de laboratorio postratamiento sugieren un resultado favorable del mismo, lo cual apoyaría las observaciones hechas por los padres de algunos pacientes que refieren mejoría clínica.

\section{SUMMARY}

A large group of patients with low extremity deformities (genu varum, genu valgun or both) was detected in the northern area of the Cauca department. Those deformities resembled a rickets picture, both clinically and radiologically. Most of them came from a semi-isolated populationgroup of Afro-mestizo ancestry, located in La Toma county, Suárez municipality.

Comparative biochemical studies between 64 patients and 109 sane subjects (first degree relatives) showed aslighthypocalcemia, increased seric phosphorus levels, a markedly high alkaline phosphatase titers, normal protein and albumin values, and high parathormone seric titers. Twentyfour hour urinalysis showed hypocalciuria and hypophosphaturia.

$25(\mathrm{OH}) \mathrm{D}_{3}$ and $1 \alpha, 25(\mathrm{OH})_{2} \mathrm{D}_{3}$ seric titers were determined to establish rickets type. There was a marked increase of $1 \alpha, 25(\mathrm{OH})_{2} \mathrm{D}_{3}$, allowing us to conclude that it corresponded to vitaminD- dependet-rickets, type II. The latter is an autosomal recessive genetically transmitted rickets with clinical and laboratory manifestations due to a defect in the vitaminD receptor.

One hundred and fifteen patiens started therapy with $1 \alpha, 25(\mathrm{OH})_{2} \mathrm{D}_{3}(0.50 \mu \mathrm{g} / \mathrm{d})$, calcium $(1,840$ $\mathrm{mg} / \mathrm{d})$, phosphorus $(1,424 \mathrm{mg} / \mathrm{d})$ given in two doses per day. Serum titers of 21 patients were analysed before and after treatment, and values tended to become normal. Molecular studies, mRNA and cDNAvitamin D receptor gen, obtained from fibroblast cultures from two patients with severe compromise, showed a normal nucleotide sequence.

A comparison between clinical and laboratory findings of the 20 reported cases in the literature and our 64 cases was done; we emphasize our observations, including the early onset of the clinical picture, pain or muscular weakness absence, without baldness, normal phosphorus values and a slight secundary hyperparathyroidism, which indicates the great heterogeneity of this particular type of rickets.

\section{AGRADECIMIENTOS}

Agradecemos a los pacientes, a sus familiares, a los miembros de la comunidad del corregimiento de La Toma, al Centro Hospital de Suárez, al Servicio de Salud del Cauca, a las autoridades del municipio de Suárez y a los funcionarios de la CVC por su colaboración y comprensión durante las muchas visitas realizadas a la región.

Los autores están especialmente agradecidos con los siguientes doctores: Mark Hughes, del Laboratorio de Diagnóstico Molecular de la Escuela de Medicina de Baylor, Houston E.U.A. por su colaboración en el clonaje y secuenciación del gen del receptor de la vitamina $D$ y por haber entrenado en estas técnicas a uno de los autores (LFG); Graham H. Beastall del Instituto de Bioquímica de Glasgow, Gran Bretaña, por la determinación de la $25(\mathrm{OH}) \mathrm{D}_{3}$ y la $1,25(\mathrm{OH})_{2} \mathrm{D}_{3}$ de los primeros pacientes evaluados; Joseph Zerwekh del Centro Médico del Suroeste de la Universidad de Dallas, E.U.A., por la determinación de la $25(\mathrm{OH}) \mathrm{D}_{3}$ y la $1,25(\mathrm{OH})_{2} \mathrm{D}_{3}$, la 24,25 hidroxilasa, 
calcitonina y parathormona en un numeroso grupo de pacientes y por el entrenamiento en las técnicas de determinación de esas sustancias a uno de los autores (MP); Marcos Danon del Hospital General de Massachusestts, por colaborarnos en lograr la determinación de la paratohormona; Harald Jueppner de laUnidad Endocrina del Hospital General de Massachussetts de Boston, EEUU, por la determinación de paratohormona en un numeroso grupo de pacientes y el entrenamiento en la determinación de esta hormona a uno de los autores (DF); a los miembros de Grupo de Sanidad del Ambiente del INS, por la determinación de fitatos y otros contaminantes ambientales.

También agradecemos muy especialmente al dr. Mauricio Restrepo por la evaluación epidemiológica inicial y a la estadística Diana Estupiñán, exfuncionaria de la División de Investigaciones Especiales del INS por los análisis estadísticos iniciales.

\section{REFERENCIAS}

1. McKusick VA. Mendelian inheritance in man. A catalogue of dominant, recessive and $X$ linked phenotypes. Ninth edition. Baltimore. The Johns Hopkins University Press, 1990; 1527-1529.

2. Fraser D, Kooh SW, Kind HP, et al. Pathogenesis of hereditary vitamin-D-dependent rickets: an inborn error of vitamin D metabolism involving defective conversion of 25-hydroxivitamin D to 1 $\alpha, 25$-dihydroxivitamin $D$. New Eng J Med 1973; 289:817.

3. Brooks $\mathrm{MH}$, Bell NH, Love L, et al. Vitamin D dependent rickets type II: resistance of target organs to 1,25dihydroxyvitamin D. New Eng J Med 1978; 298:996.

4. Lawson DEM, Fraser DR, KodicekE, etal. Identification of 1,25-dihydroxicholecalciferol, a new kidney hormone controling calcium metabolism. Nature 1971;230:220.

5. DeLuca HF. The vitamin D story: a collaborative effort of basic sciene and clinical medicine. FASEB J 1988; 2:224.

6. Feldman D, Chen T, Cone C, et al. Vitamin D resistant rickets with alopecia: cultured skin fibroblasts exhibit defective cytoplasmic receptors and unresponsiveness to $1,25(\mathrm{OH})_{2} \mathrm{D}_{3}$. J Clin Endocrinol Metab 1982; 55:1020.

7. Liberman UA, Halabe A, Samuel R, et al. End-organ resistance to 1,25-dihydroxycholecalciferol. Lancet $1980 ; 1: 504$.

8. Griffin JE, Zerwekh JE. Impaired stimulation of 25- hydroxivitamin D-24- hydroxilase in fibroblast from a patient with vitamin D dependent rickets, type II. A form of receptor-positive resistance to 1,25-dihydroxyvitamin D3. J Clin Invest. 1983; 72:1190.

9. Reichel H, Koffler HP, Norman AW. The role of vitamin $D$ endocrine system in health and disease. New Eng J Med. 1989; 320:980.

10. Gamblin GT, Liberman UA, Eil C, et al. Vitamin D dependent rickets type II: defective induction of 25hydroxyvitamin $\mathrm{D}_{3}$-24-hydroxylase by 1,25dihydroxyvitamin $D_{3}$ in cultured skin fibroblasts. J Clin Invest 1985; 75:954.

11. Rigby WFC, Stacy T, Fanger MW. Inhibition of T lymphocytes mitogenesis by 1,25-dihydroxyvitamin $D_{3}$ (calcitriol). J Clin Invest 1984; 74:1451.

12. Sone T, Scott RA, Hughes MR, et al. Mutant vitamin $D$ receptors wich confer hereditary resistance to 1,25hydroxivitamin $D_{3}$ in humans are transcriptionally inactive in vitro. J Biol Chem1989; 264:20230.

13. Liberman UA, Eil C, Marx SJ. Resistance to 1,25dihydroxyvitamin D. J Clin Invest. 1983; 71:192.

14. Evans RM. The steroid and thyroid hormone receptor superfamily. Science 1988; 240:889.

15. McDonnell DP, Mangelsdorf DJ, Pike JW, et al. Molecular cloning of complementary DNA, the avian receptor of Vitamin D. Science 1987; 235:1214.

16. Baker AR, McDonnell DP, Hughes M, et al. Cloning and expression of full length CDNA encoding human vitamin D receptor. Proc Natl Acad Sci 1988; 85:3294.

17. Hughes MR, Malloy PJ, Kieback DG, et al. Point mutations in the human vitamin $D$ receptor gene associated with hypocalcemic rickets. Science 1988 ; 242:1702.

18 Pike J.W. Molecular mechanisms of cellular response to the vitamin D3 hormone. Chapter 8, In: Coe F Favus M. Disorders of bone and mineral metabolism. Houston, Texas 1992: 163-194

19 Malloy PJ, Hochberg Z, Tiosano D, et al. The molecular basis of hereditary 1,25-dihydroxyvitamin $D_{3}$ resistant rickets in seven related families. J Clin Invest 1990; 86:2071.

20. Saijo $\mathbf{T}$, Ito $\mathrm{M}$, Takeda $\mathrm{E}$, et al. A unique mutation in the vitamin $D$ receptor gene in three Japanese patients with vitamin D-dependent rickets type II: utility of single-strand conformation polymorphism analysis for heterozygous carrier detection. Am J Hum Genet 1991; 49:668.

21. Kristjansson K, Rut AR, Hewison M, et al. Four novel mutations in the vitamin $D$ receptor gene of patients with hereditary vitamin D resistant rickets. Am 
J Hum Genet 1992; 51:A131.

22. Colmenares G. Historia económica y social de Colombia. Popayán: una sociedad esclavista 1680 1800. Bogotá Ediciones la Carreta, 1979.

23. Reinhart TA, Horst RL, Orf JW, et al. A microassay for 1.25 dihydroxivitamin $D$ not requiring $\mathrm{HPLC}$ : application to clinical studies. J Clin Endocrinol Metab. $1984 ; 58: 91$

24. Bermúdez A, Pineda $\mathbf{M}$. Técnicas de tamizaje de errores congénitos del metabolismo. Manual de procedimientos. Bogotá: Red Nacional de Laboratorios. Instituto Nacional de Salud, 1988:13-19.

25. Randerath, K. Cromatografia en capa fina. Urmo, enciclopedia de la química industrial. Tomo 8, Bilbao, 1970.

26. Kusma J. Basic statistics for the health sciences. First edition. USA Mayfield publishing company 1984.

27. Weatherall DJ, Clegg JB, Higgs DR. et al. The Hemoglobinopathies. Chapter 93 In Scriver Ch, Beaudet A. Sly $W$, et al. The metabolic basis of inherited disease. Sixth edition USA McGraw Hill 1989; 10291044.

28. Marx SJ. Vitamin D and other calciferols, Chapter 80 , In Scriver $\mathrm{CH}$, Beaudet A, Sly W, et al. The metabolic basis of inherited disease. Sixth edition, USA, McGraw Hill 1989.

29. Behrman R, Vaughan V. Nelson. Tratado de pediatria. Edit Interamericana McGraw-Hill. Tomo I, 13a edición México.1987; 2029-2045.

30. Marx S,Spiegel A, Brown E, et al. A familial syndrome of decrease in sensitivity to 1,25 -dihidroxyvitamin $D$. $\mathrm{J}$ Clin Endocrinol Metab 1978; 47:1303.

31. Rosen J, Fleischman A, Finberg L, et al. Rickets with alopecia: an inborn error of vitamin D metabolism. J Pediatrics 1979; 729.

32. Zerwekh J, Glass K, Jowsey J, et al. An unique form of osteomalacia associated withend organ refractoriness to 1,25-dihydroxyvitamin D and apparent defective synthesis of 25-hydroxyvitamin D.J Clin Endocrinol Metab 1979; 49:171.

33. Liberman U, Samuel R, Halabe A,et al. End-organ resistance to 1,25-dihydroxycholecalciferol. 1980:504.

34. Kudoh $\mathbf{T}$, Kumagai $\mathrm{T}$, Uetsuji $\mathbf{N}$, et al. Vitamin $\mathrm{D}$ dependent rickets: decreased sensitivity to 1,25 -

dihydroxyvitamin D. Eur J Pediatr 1981: 137:307.

Feldman D, Chen $\mathrm{T}$, Cone $\mathrm{CH}$, et al. Vitamin $\mathrm{D}$ resistant rickets with alopecia: cultured skin fibroblasts exhibit defective cytoplasmic receptors and unresponsiveness to 1,25(OH)2D3. J Clin Endocrinol Metab 1982; 55:1020.

36. Balsam S, Garabedian M, Liberman U, et al. Rickets and alopecia with resistance to 1.25-dihydroxyvitamin D: two different clinical courses with two different cellular defects. J Clin Endocrinol Metab 1983: 57:803.

37. Hochberg Z, Benderli A, Levy J, et al. 1,25dihydroxyvitamin $\mathrm{D}$ resistance rickets, and alopecia. Am J Med 1984; 77:805.

Hirst M, Hochman H, Feldman D, et al. Vitamin D resistance and alopecia: a kindred with normal 1,25dihydroxyvitamin $D$ binding, but decreased receptor afinity for deoxyribonucleic acid. J Clin Endocrinol Metab 1985; 60:490.

39 Malloy P, Hochberg Z, Pike J, et al. Abnormal binding of Vitamin $D$ Receptors to deoxyribonucleic acid in a kindred with vitamin D-dependent rickets, Type II. J Clin Endocrinol Metab 1989; 68:263.

40 Takeda E, Yokota I, Kawakami I, et al. Two siblings with vitamin-D-dependent rickets type II: no recurrence of rickets for 14 years after cessation of therapy. Eur J Pediatr 1989; 149:54.

Manandhar D, Sarkawi, Hunt M. Rickets with alopecia-remission following a course of 1-alfa-hydroxy vitamin D3 therapy. Eur J Pediatr 1989; 761.

McKusick VA. Medical genetic studies of the Amish. Baltimore and The Johns Hopkins University Press. and London: 1978.

43 Balsan S, Garabedian M, Larchet M, et al. Longterm nocturnal infusions can cure ricketes and promote normal mineralization in hereditary resistance to 1,25dihydroxyvitamin D. J Clin Invest 1986; 77:1661.

44 Walters, M. Newly identified actions of the vitamin D endocrine system. Endocrine Reviews 1992; 13:719.

45 Braekeleer M, Larochelle J. Population genetics of vitamin D-dependent rickets in northeastern Quebec. Ann Hum Genet 1991; 55:283.

Iglesias A, Vásquez J, Abud C. Enfermedades metabólicas del hueso. Volúmenes I y II. Santafé de Bogotá Instituto Nacional de Salud.1992. 